\title{
Effect of Gum Chewing on Intestinal Functions after Gynecological Operations: A Randomized Controlled Study
}

\author{
Taylan ŞENOL ${ }^{1}$, Mesut POLAT ${ }^{1}$, Enis ÖZKAYA ${ }^{1}$, Gökhan ÜNVER ${ }^{1}$, Ateş KARATEKE ${ }^{1}$ \\ Istanbul, Turkey
}

\begin{abstract}
OBJECTIVE: We aimed to assess the efficacy of gum chewing on intestinal functions after gynecological operations.

STUDY DESIGN: A total of 86 women who underwent gynecological operation with different indications were randomly assigned to 2 groups: Group 1 was assigned to gum chewing after operation $(n=52)$, while Group 2 was directed to routine postoperative care $(n=52)$. Time of first bowel sound and defecation after surgery were recorded to assess the effect of gum chewing. Operation time, blood loss, type of incision, pre and postoperative serum hemoglobin levels were all evaluated.
\end{abstract}

RESULTS: There was no difference between groups in terms of age, duration of operation, intraoperative blood loss, pre and postoperative serum hemoglobin levels, duration to first bowel sound, flatulence and defecation $(p>0.05)$. Age $(r=0.234, p=0.032)$, type of incision $(r=0.228, p=0.037)$ were significantly correlated with the time to first bowel sound. Type of incision $(r=0.295, p=0.006)$, duration of operation $(r=0.277, p=0.01)$ and intraoperative blood loss $(r=0.298, p=0.006)$ were significantly correlated with the time to first flatulence. In multivariate regression analyses, none of the variables were found to be significant parameter for time to first bowel sound ( $p>0.05)$.

CONCLUSION: Gum chewing does not affect some of the gastrointestinal functions after gynecological operations and there is no single parameter for time to first bowel sound, first flatulence and first defecation, individual surgical and medical condition differences should be kept in mind while evaluating intestinal functions.

Keywords: Gum chewing, Bowel function, Gynecological operations

Gynecol Obstet Reprod Med 2016;22(3):160-164 DOI:10.21613/GORM.2016.156

\section{Introduction}

Following an abdominal surgery, a transient non mechanical blockage of small and large intestine can be seen as an important postoperative problem (1). Frequency of postoperative ileus was reported to be $10.3 \%$ after an abdominal surgery (2). Ileus is determined in case of unrecovered intestinal functions after $3^{\text {rd }}$ to $5^{\text {th }}$ postoperative day following an abdominal surgery (3). Normalization of intestinal functions can be indirectly verified by declaration of flatulence and defecation by the patient (4). Recovery of intestinal function decreases postoperative hospitalization length and abdominal tenderness, also provides earlier oral intake (5). Decreased length of hospitalization lead to reduction in the rates of hospital infections, risk of deep vein thrombosis and the cost (6). Pathogenesis of ileus is multifactorial and several factors have been identified

\footnotetext{
${ }^{1}$ Zeynep Kamil Training and Research Hospital Obstetric and Gynecology Department, Istanbul

Address of Correspondence: Taylan Şenol

Opr.Dr. Burhanettin Üstünel Cad. No:10

Üsküdar, Istanbul, Turkey

taylan_senol@hotmail.com

Submitted for Publication:

02.05 .2016

Accepted for Publication:

06.09 .2016
}

for ileus development. Opioid treatment during surgery and severe visceral manipulations may also increase the risk of ileus $(7,8)$. On the other hand, a dysfunctional parasympathetic activity may be the underlying abnormality (6). In order to prevent postoperative ileus, some interventions have been proposed to be used, including appropriate analgesic use, epidural anesthesia, gum chewing, laparoscopic surgery, metoclopramide, erythromycin, neostigmine, alvimopan administration, nasogastric decompression, intravenous fluid infusion and early enteral feeding $(6,9)$. Gum chewing has been shown to be effective to prevent postoperative ileus after cesarean section (10). However, there is not enough number of randomized studies or meta-analyses to assess the effectiveness of gum chewing after gynecologic operations (11). In this study, we aimed to evaluate the effectivity of gum chewing on intestinal functions after gynecological operations in a randomized manner.

\section{Material and Method}

Between February 2015 and August 2015, totally 104 women who underwent gynecological operations with different indications in Department of Obstetrics and Gynecology in Zeynep Kamil Women and Children's Health Training and 
Research Hospital were randomly assigned into 2 groups: Group 1 was assigned to gum chewing $(\mathrm{n}=52)$, while Group 2 was directed to routine postoperative care without chewing $(\mathrm{n}=52)$. Randomization was performed by using computer program (Clinical trials registration number: NCT02455739). Flow chart of the study population was shown in Figure 1. Sample size was calculated according to the study by Ledari et al with $95 \%$ confidence interval and $80 \%$ statistical power (12, Epiinfo). Study protocol was approved by the Zeynep Kamil Women and Children's Health training and Research Hospital Ethics committee (Approval number: 34/2015) and signed informed consent was obtained from each participants. Time of first bowel sound after surgery first flatulence and first defecation were recorded to assess the effect of gum chewing. Operation time, intraoperative blood loss, type of incision, pre and postoperative serum hemoglobin levels were all evaluated. Eligible women for gum chewing after gynecologic surgery were included in the study. Exclusion criteria were previous gastrointestinal intervention, previous abdominal surgery, history of drug consumption, water and electrolyte disturbances, pancreatitis or peritonitis, no willingness to cooperate, inability to chew gum, intra- and post-operative complications. hypothyroidism, and muscular or neurological disorders. Incision types were divided into three groups as; sub umbilical midline vertical and sub or supra umbilical midline vertical incisions. Age, body mass index, indication of surgical intervention, pre and postoperative blood cell counts, type of surgical intervention, duration of operation (minutes), intraoperative blood loss were all recorded for each participant. Body mass index was calculated by the formula: Weight/height2, duration of operation was calculated as minutes from first incision to fascial closure by stop watch. Blood loss was calculated by the sum of $60 \mathrm{ml}$ per small soaked swab $(10 \times 10 \mathrm{~cm})$, $350 \mathrm{~mL}$ per large soaked swab $(45 \times 45 \mathrm{~cm})$ and volume in reservoir of surgical aspirator. Study group was prescribed the sugar-free gum after recovery from anesthesia three times a day, for each time subjects chewed gum for one hour, until first bowel sound detected by auscultation. Data was collected by questionnaires, and the physical examination of subjects. All patients were requested to mention the time of the first bowel movement, passage of flatus and defecation. An independent medical staff who was unaware of the study protocol, visited the patients for every hour, and recorded. Groups were compared in terms of time to first bowel sound, flatus and defecation to determine the effect of gum chewing on gastrointestinal functions after gynecological operations. Patients were diagnosed as sub ileus who did not experience flatus within the first 24 hours of postoperative period (13).

\section{Statistical Analyses}

All data was entered to SPSS version 15 software for Windows (SPSS, Chicago, IL), descriptive analyses were used to show minimum, maximum and the mean levels, student- $t$ test was used to compare means between two groups, correlation analyses was used to show degree of correlations between variables and multivariate analyses was used to show adjusted associations. A p value of less than 0.05 was considered statistically significant.

\section{Results}

Clinical and demographic characteristics of the study population were summarized in Table 1 and flow chart of the study was illustrated in Figure 1. There was no difference between groups in terms of age, duration of operation, blood loss, pre and postoperative serum hemoglobin levels, time to first bowel sound, flatulence and defecation $(\mathrm{p}>0.05)$. There was no significant difference between groups in terms of systemic disorder, type of incision and the indication of surgery $(\mathrm{p}>0.05)$ (Table 2,3). Age $(\mathrm{r}=0.234, \mathrm{p}=0.032)$, type of incision $(\mathrm{r}=0.228, \mathrm{p}=0.037)$ were significantly correlated with the time to first bowel sound. Type of incision $(r=0.295, p=0.006)$, duration of operation $(r=0.277, \mathrm{p}=0.01)$ and blood loss $(\mathrm{r}=0.298$, $\mathrm{p}=0.006$ ) were significantly correlated with the time to first flatulence. In multivariate regression analyses, none of the variables were found to be significant parameter for time to first bowel sound $(p>0.05)$. Also none of the variable was found to affect time to first flatulence $(\mathrm{p}>0.05)$. There were 11 $(31.4 \%)$ cases of sub ileus in study group, in the control group $19(37.3 \%)$ cases were noted ( $p>0.05)$. No ileus case was observed in the groups.

Table 1: Comparison of the two groups with regard to some clinical and demographic characteristics

\begin{tabular}{lccc}
\hline & $\begin{array}{c}\text { Control }(\mathrm{n}=51) \\
\text { Mean } \pm \text { SD }\end{array}$ & $\begin{array}{c}\text { Chewing Gum }(\mathrm{n}=35) \\
\text { Mean } \pm \text { SD }\end{array}$ & $\mathrm{NS}$ \\
\hline Age (year) & $46.9 \pm 11.5$ & $45.6 \pm 8.8$ & $\mathrm{NS}$ \\
$\mathrm{BMI}\left(\mathrm{kg} / \mathrm{m}^{2}\right)$ & $28.2 \pm 5.1$ & $29.5 \pm 5.2$ & $\mathrm{NS}$ \\
Preop Hb $(\mathrm{gr} / \mathrm{dL})$ & $12.1 \pm 1.3$ & $12.1 \pm 1.3$ & $\mathrm{NS}$ \\
Postop Hb(gr/dL) & $10.7 \pm 1.4$ & $10.4 \pm 1.1$ & $\mathrm{NS}$ \\
Blood loss $(\mathrm{mL})$ & $230.4 \pm 249$ & $277.1 \pm 253$. & $\mathrm{NS}$ \\
Bowel sound (hour) & $6.08 \pm 1.8$ & $5.59 \pm 1.8$ & $\mathrm{NS}$ \\
First flatulence (hour) & $23.5 \pm 10.7$ & $23.7 \pm 13$ & $\mathrm{NS}$ \\
Defecation time (hour) & $47.9 \pm 20.9$ & $45.6 \pm 18.7$ & \\
\hline
\end{tabular}

BMI: Body mass index, NS: Non significant 


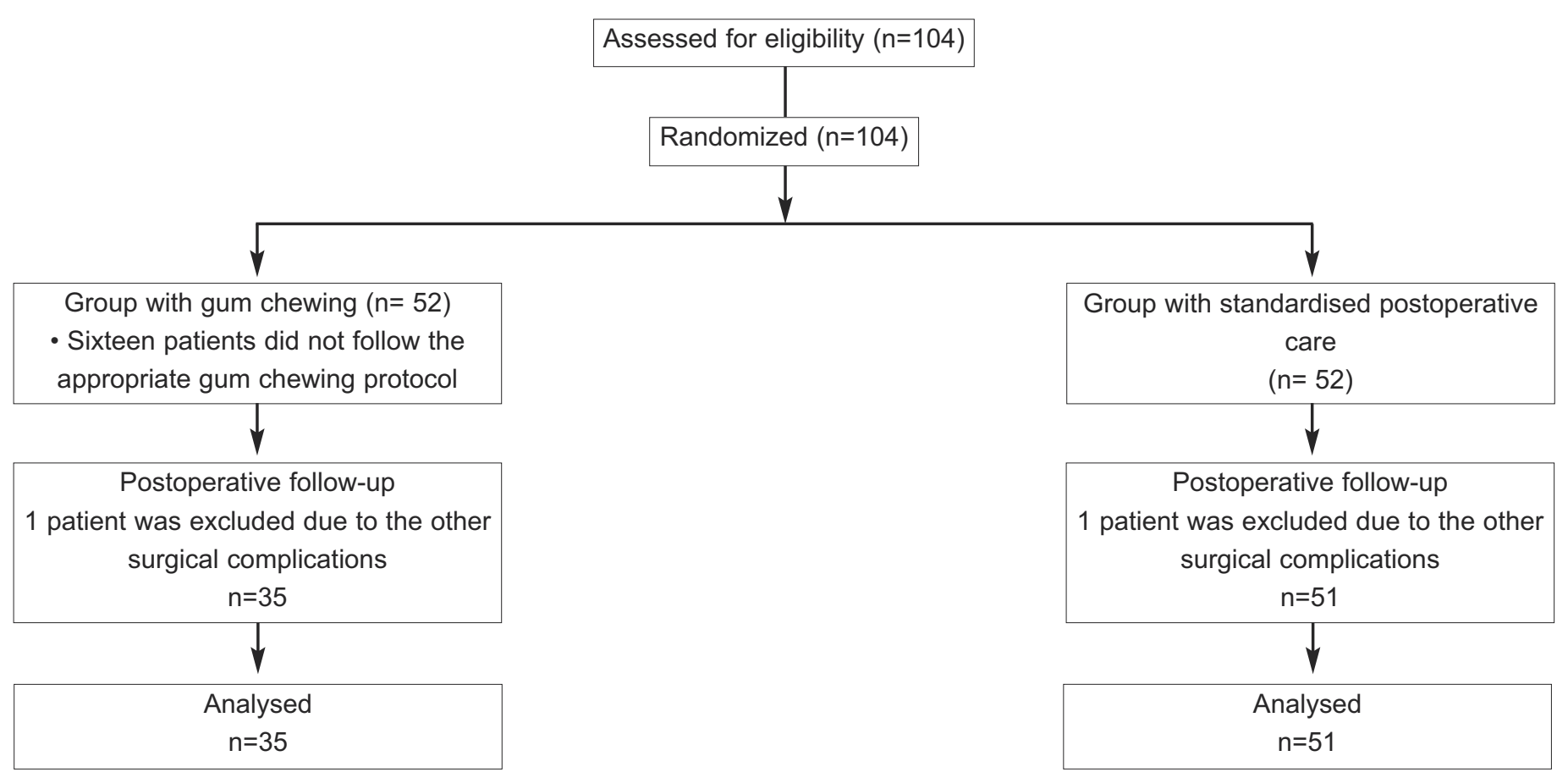

Figure 1: Flowchart of patients through the trial.

Table 2: Summary and comparison of type of surgeries between the groups

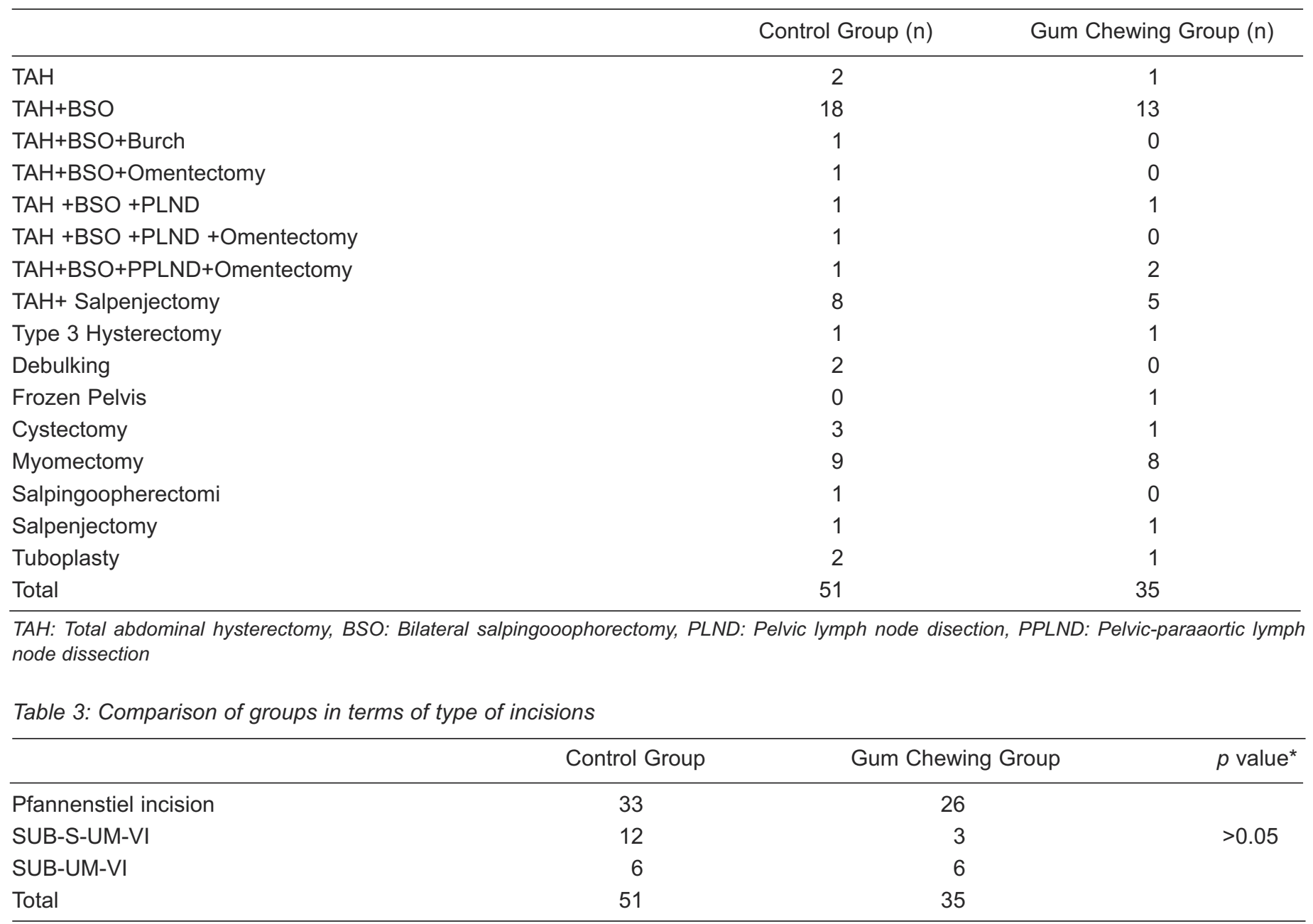

Chi square test $p<0.05$, SUB -S-VI: Sub umblical and supra umblical vertical incision, SUB-UM-VI: Sub umblical vertical incision 


\section{Discussion}

Our data showed no difference between with or without gum chewing groups in terms of age, body mass index, duration of operation, pre and postoperative blood counts. Previous studies also compared groups for age, body mass index and the duration of operation $(3,14,15)$. Additionally, previous studies showed the importance of surgical manipulation on postoperative intestinal functions $(10,12,16)$. Generally, it is not expected to experience too much surgical manipulation during cesarean section. We conducted this study in a study population undergoing gynecological operations. Although we studied a heterogeneous group of surgeries, randomization and regression analyses helped us to overcome this drawback, so that we obtained an opportunity to assess the effect of type of surgical intervention and the incision. In our institution most of the time, ileus cases are observed among patients who underwent gynecological surgery rather than cesarean section, so in order to represent the whole population, we established a sample including different types of surgical interventions. We thought that, this sample is an appropriate reflection of our routine gynecological practice.

In our study indications of surgeries, rate of systemic disorders and type of incisions were similar between groups. According to previous studies, gum chewing was found to be effective to decrease time to first bowel sound, flatulence and the defecation after gastrointestinal surgeries (17-19). Safety and effectiveness of gum chewing in decreasing postoperative ileus and nausea have been shown in a previous study (15). However, our results did not show significant difference between groups in terms of time to first flatus and bowel movements. Gum chewing was also found to be beneficial on bowel movements after laparoscopic surgeries where the probability of manipulation was minimal (20).

In our study, no difference was observed between groups in terms of time to first bowel sound, flatulence and defecation, however, there was a significant correlation between the type of incision, duration operation, blood loss and the time to first flatulence. Negative effect of increased catecholamine, and, surgical manipulations on pacemakers, electrolyte imbalances, peritoneal irritations and analgesic substances have been proposed to be some of the factors that lead to intestinal malfunction as pathophysiological mechanism of postoperative ileus (21-23) furthermore elevated concentrations of some vasoactive peptides has been also proposed to have inhibitory effect on smooth muscle contractions in some studies (24), these data explain why we found a significant correlation between aforementioned variables. Blood loss and surgical stress may also be major causes of catecholamine discharge and electrolyte imbalance which were shown to be main contributors for postoperative ileus.

\section{Conclusion}

Gum chewing does not affect gastrointestinal functions after gynecological operations and there is no single parameter for time to first bowel sound, first flatulence and first defecation. Further investigations are needed and individual surgical and medical condition differences should be kept in mind while evaluating intestinal functions.

\section{References}

1. Vather R, Bissett I. Management of prolonged post-operative ileus: evidence based recommendations. ANZ J. Surg 2013;83(5):319-24.

2. Guay J, Nishimori M, Kopp S. Epidural local anaesthetics versus opioid-based analgesic regimens for postoperative gastrointestinal paralysis, vomiting and pain after abdominal surgery: A Cochrane Review. Anesth Analg 2016;123 (6):1591-1602.

3. Ghafouri A, Soroush AR, Moeini N, Hedayat A, Khourgami ZH. The efficacy of sugar free gum chewing after upper gi tract operation on ileus: a clinical trial. Iranian J Surg 2008;16(1):79-84.

4. Steed HL, Capstick V, Flood C, Schepansky A, Schulz J, Mayes DC. A randomized controlled trial of early versus "traditional" postoperative oral intake after major abdominal gynecologic surgery. Am J Obstet Gynecol 2002; 186(5):861-5.

5. Akhlaghi F, Pourjavad M, Mansouri A, Tara F, Vahedian M. Effect of Gum Chewing on Prevention of Post Cesarean Ileus. Hayat 2008;14(2):35-40.

6. Johnson MD, Walsh RM. Current therapies to shorten postoperative ileus. Cleve Clin J Med 2009;76(11):64-8.

7. Bonventre S, Inviati A, Di Paola V, Morreale P, Di Giovanni S, Di Carlo P, et al. Evaluating the efficacy of current treatments for reducing postoperative ileus: a randomized clinical trial in a single center. Minerva Chirurgica 2014;69(1):47-55.

8. Le Blanc-Louvry I, Costaglioli B, Boulon C, Leroi AM, Ducrotte P. Does mechanical massage of the abdominal wall after colectomy reduce postoperative pain and shorten the duration of ileus? Results of a randomized study. J Gastro Surg 2002;6(1):43-9.

9. Jorgensen H, Wetterslev J, Moiniche S, Dahl JB. Epidural local anaesthetics versus opioid-based analgesic regimens on postoperative gastrointestinal paralysis, PONV and pain after abdominal surgery. Cochrane Database Syst Rev 2000;(4):CD001893.

10. Short V, Herbert G, Perry R, Atkinson C, Ness AR, Penfold $\mathrm{C}$, et al. Chewing gum for postoperative recovery of gastrointestinal function. Cochrane Database Syst Rev 2015;20:2.

11. Ledari FM, Barat S, Delavar MA, Banihosini SZ, Khafri S. Chewing sugar-free gum reduces ileus after cesarean section in nulliparous women: a randomized clinical trial. Iran Red Crescent Med J 2013;15(4):330-4.

12. Vather R, Trivedi S, Bissett I. Defining postoperative 
ileus: results of a systematic review and global survey. J Gastrointest Surg 2013;17(5):962-72.

13. Yaghmaei M, Arbabi Kalati F, Mokhtari M, Behzadian A. Comparison of oral intake profiles at 2 and 8 hours following cesarean section under spinal anesthesia. Zahedan J Res Med Sci 2010;11(4):43-51.

14. Pearl ML, Frandina M, Mahler L, Valea FA, DiSilvestro PA, Chalas E. A randomized controlled trial of a regular diet as the first meal in gynecologic oncology patients undergoing intraabdominal surgery. Obstet Gynecol 2002; 100(2):230-4.

15. Jernigan AM, Chen CCG, Sewell C. A randomized trial of chewing gum to prevent postoperative ileus after laparotomy for benign gynecologic surgery. Int J Gynecol Obst 2014;127(3):279-82.

16. Kafali H, Duvan CI, Gözdemir E, Simavli S, Onaran Y, Keskin E. Influence of gum chewing on postoperative bowel activity after cesarean section. Gynecol Obstet Invest 2010;69(2):84-87.

17. Fitzgerald JEF, Ahmed I. Systematic review and metaanalysis of chewing-gum therapy in the reduction of postoperative paralytic ileus following gastrointestinal surgery. World J Surg 2009;33(12):2557-66.

18. Chan MK, Law WL. Use of chewing gumin reducing pos- toperative ileus after elective colorectal resection: a systematic review. Dis Colon Rectum 2007;50(12):2149-57.

19. Noble EJ, Harris R, Hosie KB, Thomas S, Lewis SJ. Gum chewing reduces postoperative ileus? A systematic review and meta-analysis. Int J Surg 2009;7(2):100-5.

20. Husslein H, Franz M, Gutschi M, Worda C, Polterauer S, Leipold H. Postoperative gum chewing after gynecologic laparoscopic surgery: a randomized controlled trial. Obstet Gynecol 2013;122(1):85-90.

21. Jepsen S, Klaerke A, Nielsen PH, Simonsen O. Negative effect of Metoclopramide in postoperative adynamic ileus. A prospective, randomized, double blind study. Br J Surg 1986;73(4):290-1.

22. Peeters T, Matthijs G, Depoortere I, Cachet T, Hoogmartens J, Vantrappen G. Erythromycin is a motilin receptor agonist. Am J Physiol 1989;257(3):470-4.

23. Basse L, Hjort Jakobsen D, Billesbølle P, Werner M, Kehlet $\mathrm{H}$. A clinical pathway to accelerate recovery after colonic resection. Ann Surg 2000;232(1):51-7.

24. Carr CS, Ling KD, Boulos P, Singer M. Randomised trial of safety and efficacy of immediate postoperative enteral feeding in patients undergoing gastrointestinal resection. BMJ 1996;312(7035):869-71. 